\title{
A GENERALIZED EARNINGS-BASED STOCK VALUATION MODEL
}

\author{
by \\ MING DONG \\ Schulich School of Business, York University, Toronto \\ and \\ DAVID HIRSHLEIFER* \\ Fisher College of Business, Ohio State University
}

\begin{abstract}
This paper provides a model for valuing stocks that takes into account the stochastic processes for earnings and interest rates. Our analysis differs from past research of this type in being applicable to stocks that have a positive probability of zero or negative earnings. By avoiding the singularity at the zero point, our earnings-based pricing model achieves improved pricing performance. The out-of-sample pricing performance of the generalized earnings valuation model (GEVM) and the Bakshi and Chen pricing model are compared on four stocks and two indices. The generalized model has smaller pricing errors and greater parameter stability. Furthermore, deviations between market and model prices tend to be mean-reverting using the GEVM model, suggesting that the model may be able to identify stock market misvaluation.
\end{abstract}

\section{Introduction}

Recent research has pointed the way to an exciting new direction for the theory of stock valuation. Moving beyond traditional dividend discounting models, dynamic models have emerged that provide greater structure by assuming explicit stochastic processes for the variables that influence stock value (see, for example, Berk et al., 1999, Ang and Liu, 2001, Bakshi and Ju, 2002). Among these, a distinctive feature of the Bakshi and Chen (2001) stock valuation model (hereafter the $\mathrm{BC}$ model) is its focus on earnings. The key underlying valuation variables in this model are the level of earnings, the growth rate of earnings and the interest rates used to discount earnings. Since earnings performance is a key fundamental underlying stock prices, the $\mathrm{BC}$ model has laid out a parsimonious framework of dynamic stock valuation. Indeed, by modeling the stochastic process for earnings, and by adopting a stochastic pricing-kernel process that is consistent with the Vasicek (1977) term structure of interest rates, the $\mathrm{BC}$ model provides a closed-form solution for the stock price. Such structural modeling of the relation between

\footnotetext{
*We thank Peter Easton, Bob Goldstein, Andrew Karolyi, Anil Makhija, John Persons, Jay Ritter, René Stulz and especially Zhiwu Chen, seminar participants at Ohio State University, York University and the 2001 Northern Finance Association meetings in Halifax, Canada, for very helpful comments.
} 
stock value, earnings and interest rates offers the potential for more accurate predictions about stock prices and price movements than have been achieved previously in the literature.

Although the BC model makes important headway in modeling how stocks are priced, a property of the model that limits its applicability is that, strictly speaking, it cannot value stocks that have a positive probability of realizing zero or negative earnings. In reality, stocks that will achieve positive earnings with certainty forever do not, of course, exist. In this sense the model necessarily provides an approximation that becomes less exact the greater the likelihood that the firm will have zero or negative earnings. Indeed, as we will discuss in more depth, the $\mathrm{BC}$ model becomes extremely inaccurate when this likelihood is large.

To get a sense for how restrictive the non-negativity constraint is in practice, consider a sample of 6262 I/B/E/S-covered stocks during July 1976 and March 1998. On average, for any stock at any point in time, the chance of having a negative earnings per share number is 11.9 per cent, and 41.3 per cent of the stocks have at least one non-positive earnings outcome. This means that the $\mathrm{BC}$ model cannot be applied to over 40 per cent of the stocks for at least some period of time. In a recent study, Kothari et al. (2005) document that aggregate earnings become negative in 1993, and that portfolio average earnings are negative during some periods for portfolios of small firms and high book-to-market stocks. Of course, even the stocks which did not realize negative earnings ex ante had some probability of negative earnings.

Indeed, this constraint of the model reduces its applicability for some of the stocks that are most interesting from a valuation perspective- those that are generating relatively low earnings now, and might be in deep trouble or on the other hand may have excellent growth opportunities. Assuming a zero probability of negative earnings is also likely to be especially unrealistic for stocks that are highly volatile or are highly sensitive to systematic return factors. Since it is especially desirable for investors to have a good model for valuing speculative stocks, it is interesting to consider possible ways of generalizing the model. Indeed, another shortcoming of the BC model is that the model prices of stocks tend to fluctuate quite a lot over time, sometimes ranging wildly over a period of months. It is possible that these swings are related to difficulties in valuing speculative stocks.

This paper provides a more general stock valuation model that is not restricted to positive-earnings stocks. The ability to price stocks with nonpositive earnings is important for the model be of practical use. In fact, we will show that the problem of excluding negative earnings in the $\mathrm{BC}$ model and the problem of rapid swings in valuations are closely related. The generalized model that we provide turns out to have better pricing performance, in a sense to be made more precise below, even for stocks with positive earnings. 
From a conceptual viewpoint, the $\mathrm{BC}$ model profoundly improves on the traditional dividend discount models (DDM). The simplest form of the DDM is the Gordon (1962) model, which assumes a constant dividend growth rate and a constant discount rate for a stock. The $\mathrm{BC}$ model generalizes this classic Gordon model in several dimensions.

First, dividend growth is allowed to be stochastic. Second, the stock price is linked to the firm's underlying earnings rather than dividends. Firms' dividend policies are typically sticky, and many firms choose not to pay cash dividends, so earnings are far more informative. ${ }^{1}$ Specifically, the $\mathrm{BC}$ model assumes earnings and earnings growth processes that allow changing longrun earnings growth rate to reflect the business conditions and characteristics of the firm. Third, instead of non-stochastic interest rates, the interest rate movement is characterized by a mean-reverting stochastic process. Finally, the BC model adopts a stochastic pricing kernel which ensures that the model is arbitrage-free as in Harrison and Kreps (1979).

In consequence of these generalizations, the $\mathrm{BC}$ model possesses a rich structure characterized by a set of parameters reflecting the macroeconomic and firm-specific environment. Stocks with similar accounting and earnings forecast data may be valued quite differently by the market, because they may be at different stages of their industry business cycles or may be perceived by the market as being managed by executives of different quality. These factors can potentially be captured by the parameters of the model, when these parameters are backed up by previous market price data. For example, a well-managed firm will be highly valued by the market, and this can cause structural parameters to set the model's valuation higher for given earnings data.

A highly influential stock valuation model developed in the accounting literature and applied in both accounting and finance is the residual income model (see Ohlson, 1995). The residual income model, which relates stock price to book value of equity and 'residual income', can be viewed as a variant of the DDM with some assumptions about the relations among accounting numbers, particularly the 'clean surplus' relation. ${ }^{2}$ The residual income model is easily implementable, and incorporates the changing business operations of the firms into valuation by using earnings forecasts (either from financial analysts or from researchers' own projection) as part of the model inputs. Empirical studies by Frankel and Lee (1998) and Lee et al. (1999) show that, with a multi-stage residual income model, one can achieve a better pricing fit than the traditional DDM as well as a better return pre-

${ }^{1}$ See, for example, Penman and Sougiannis (1998), who compare the dividend, cash flow and earnings approaches to stock valuation.

${ }^{2}$ Residual income is earnings in excess of what is required from the cost of equity. The clean surplus assumption states that dividends is earnings minus the change in book value of equity.

(C) Blackwell Publishing Ltd and The University of Manchester, 2005. 
dictive power than financial ratio analysis. Ritter and Warr (2002) show that the return predictive power of the residual income model is substantially enhanced when earnings are capitalized at the real, rather than nominal, discount rate.

However, the residual income model, as with the DDM, does not offer much structure to constrain the elements of the model. The 'intrinsic value' of the stock is calculated as the sum of the firm's book value and the sum of discounted 'residual earnings' (earnings in excess of what would be expected based upon the appropriate rate of return on the firm's capital stock, measured by book value) for each period. The lack of a parameterized structure allows valuation at a given time to be independent of how the market has valued the stock in the past, because there are not enough structural parameters that can be backed up by historical market prices to reflect how the market values the stock.

Furthermore, the residual income model often requires an empirical, $a d$ hoc estimation of the terminal value of the stock at the end of the earnings forecast period. Since such a terminal value constitutes a large proportion of the stock price, the estimate of the residual income model can be sensitive to this assumption. ${ }^{3}$ In contrast, models with explicitly defined processes for interest rates and earnings are able to use current state variables to form conditional expectations about the future values of the model inputs. As such, there is no need to arbitrarily guess the terminal value of the stock price at a certain future date. These disadvantages of the residual income model must be weighed against the fact that the $\mathrm{BC}$ model cannot be applied to negative earnings stocks owing to the assumption that earnings follow a geometric Brownian motion.

This paper introduces an alternative, more flexible earnings adjustment parameter to the BC earnings and earnings growth processes. The firm's earnings are expressed as the difference between two streams: adjusted earnings and fixed buffer earnings. The stock price is accordingly the difference of the discounted payout from these two streams. Adjusted earnings (rather than the earnings themselves) grow at an uncertain rate, and the adjusted earnings growth rate (rather than the earnings growth rate) follows a meanreverting diffusion. In consequence, negative earnings are permissible and the BC model's singularity at zero earnings is removed. We derive a closed-form solution to the generalized valuation model (hereafter, the generalized earnings valuation model, or GEVM), in which the BC model nests as a special case.

In the GEVM, the level of buffer earnings is a free parameter estimated from the time series of earnings and stock price data, together with other

\footnotetext{
${ }^{3}$ See, for example, Frankel and Lee (1998) and Lee et al. (1999) for issues related to residual income model estimation. See Ang and Liu (2001) and Bakshi and Ju (2002) for a theoretical generalization of the residual income model.
} 
model parameters. Buffer earnings can be interpreted as the firm's costs, or as some portion of these costs. If we interpret the buffer earnings as the total costs, then it follows that the adjusted earnings should be the firm's revenues. Therefore, the model can be extended to a revenues/costs-based alternative in which revenues and costs follow different stochastic processes, and the stock price is the difference of the discounted revenues and costs. We analyze how separate modeling of revenues and costs (as compared with an overall focus on earnings) changes the model, and derive a pricing formula for the revenue/cost approach to stock valuation.

In the empirical part of the paper, the pricing performance of the GEVM is compared to that of the BC model on two stock indices and four stocks. Since the BC model cannot price negative earnings stocks, to permit comparison of the two models we focus on positive earnings stocks/indices. ${ }^{4}$

The GEVM significantly reduces out-of-sample pricing error and model price variance, even though this sample consists of positive earnings stocks and indices. We also find that the GEVM mispricing (the deviation between actual and model prices) presents much stronger mean-reversion than $\mathrm{BC}$ mispricing. This is a desirable feature since it suggests that actual stock prices move toward GEVM stock prices much faster than toward BC model prices. Finally, we investigate how to interpret the model's buffer earnings and its determinants in a larger sample that contains stocks with negative earnings. We examine a set of different types of costs including total costs, R\&D, advertising and depreciation costs, and find that the level of buffer earnings is positively related to each type of cost incurred by the firm.

The remainder of the paper is organized as follows. The next section reviews the Bakshi and Chen (2001) model. Section 3 generalizes the BC model to develop the GEVM. This section also derives the earnings components valuation model based upon the revenues/costs approach to stock valuation. Section 4 presents empirical results on model performance. Section 5 concludes.

\section{The BaKshi-Chen Model}

We consider a continuous-time, infinite-horizon economy characterized by a pricing-kernel process $M(t)$. The stock price of a generic firm with an infinite dividend stream $\{D(t): t \geq 0\}$ is given by

$$
S(t)=\int_{t}^{\infty} E_{t}\left[\frac{M(\tau)}{M(t)} D(\tau)\right] \mathrm{d} \tau
$$

${ }^{4}$ Chen and Dong (2003) test the empirical performance of the GEVM on a much larger data sample. The model appears to perform equally well for stocks with positive and negative earnings.

(C) Blackwell Publishing Ltd and The University of Manchester, 2005. 
where $E_{t}(\cdot)$ is the time- $t$ conditional expectation operator with respect to the objective probability measure (Duffie (1996) describes further details as to necessary technical conditions). The existence of a pricing-kernel process $M(t)$ is also a necessary and sufficient condition for no arbitrage opportunities to be available in the economy (as in Harrison and Kreps, 1979).

The pricing kernel $M(t)$ is assumed to follow the Ito process under the true probability measure

$$
\frac{\mathrm{d} M(t)}{M(t)}=-R(t) \mathrm{d} t-\sigma_{m} \mathrm{~d} \omega_{m}(t)
$$

where $\sigma_{m}$ is a constant, and the instantaneous interest rate, $R(t)$, follows a single-factor Vasicek (1977) term structure process

$$
\mathrm{d} R(t)=\kappa_{r}\left[\mu_{r}^{0}-R(t)\right] \mathrm{d} t+\sigma_{r} \mathrm{~d} \omega_{r}(t)
$$

$\kappa_{r}, \mu_{r}^{0}$ and $\sigma_{r}$ all constant. To make the model internally consistent, the drift term of the pricing kernel $M(t)$ is set to be the negative of the instantaneous interest rate $R(t) .^{5}$ The structural parameters have the standard interpretations: $\kappa_{r}$ measures the speed at which the spot rate $R(t)$ adjusts to its longrun mean, $\mu_{r}^{0}$. This single-factor term structure is restrictive, but it could easily be generalized to a multi-factor term structure in the Vasicek or the Cox et al. (1985) class. ${ }^{6}$ The cost of doing so is that it would create many more parameters, creating a risk of noisy parameter estimates and of model overfitting. To make the model implementable, we pursue a single-factor term structure model here.

Dividend per share $D(t)$ relates to net earnings per share $Y(t)$ according to

$$
D(t)=\delta Y(t)+\varepsilon(t)
$$

where $\varepsilon(t)$ is an independently and identically distributed noise process with zero mean. This parameterization is inspired by the classic survey of Lintner (1956), which found that firms adjust toward a target dividend payout ratio.

Equation (4) can be interpreted more broadly to be meaningful even for firms that do not pay any cash dividends. Under this interpretation, regardless of whether the firm pays dividends, $D(t)$ is to be interpreted as an 'implied dividend' that is responsible for the stock price, and is simply a constant times earnings plus a noise.

The final assumptions of the model are that earnings and earnings growth follow the processes

\footnotetext{
${ }^{5} \mathrm{An}$ asset whose return is uncorrelated with the pricing kernel should earn the risk-free rate $R(t)$; see equation (25) in Section 3.2.

${ }^{6}$ Examples include Brennan and Schwartz (1979) and Longstaff and Schwartz (1992).

(C) Blackwell Publishing Ltd and The University of Manchester, 2005.
} 


$$
\begin{aligned}
\frac{\mathrm{d} Y(t)}{Y(t)} & =G(t) \mathrm{d} t+\sigma_{y} \mathrm{~d} \omega_{y}(t) \\
\mathrm{d} G(t) & =\kappa_{g}\left[\mu_{g}^{0}-G(t)\right] \mathrm{d} t+\sigma_{g} \mathrm{~d} \omega_{g}(t)
\end{aligned}
$$

where $\sigma_{y}, \kappa_{g}, \mu_{g}^{0}$ and $\sigma_{g}$ are constants.

The long-run mean for both $G(t)$ and actual earnings growth $\mathrm{d} Y(t) / Y(t)$ is $\mu_{g}^{0}$, and the speed at which $G(t)$ adjusts to $\mu_{g}^{0}$ is reflected by $\kappa_{g}$. Furthermore, $1 / \kappa_{g}$ measures the duration of the firm's business growth fluctuations. The volatility of both earnings growth and changes in expected earnings growth are time-invariant. Shocks to expected growth, $\omega_{g}(t)$, are assumed to be uncorrelated with systematic shocks $\omega_{m}(t)$, reflecting the fact that $G(t)$ is firmspecific. However, $\omega_{g}(t)$ is in general correlated with interest rate shocks $\omega_{r}(t)$, with correlation coefficient denoted by $\rho_{g, r}$. The correlations of $\omega_{y}(t)$ with $\omega_{g}(t)$, $\omega_{m}(t)$ and $\omega_{r}(t)$ are denoted by $\rho_{g, y}, \rho_{m, y}$ and $\rho_{r, y}$ respectively. The noise process $\varepsilon(t)$ in (2) is assumed to be uncorrelated with $G(t), M(t), R(t)$ and $Y(t)$.

Equations (5) and (6) offer a rich yet tractable model for the firm's earnings process. Negative earnings growth is possible, and earnings growth can be affected by both a short-run rate $G(t)$ and a long-run mean rate $\mu_{g}^{0}$. But, as we will discuss, equation (5) precludes negative earnings.

Bakshi and Chen (2001) show that under the above assumptions the equilibrium stock price $S(t)$ satisfies the following stochastic partial differential equation (PDE):

$$
\begin{aligned}
& \frac{1}{2} \sigma_{y}^{2} Y^{2} \frac{\partial^{2} S}{\partial Y^{2}}+\left(G-\lambda_{y}\right) Y \frac{\partial S}{\partial Y}+\rho_{g, y} \sigma_{y} \sigma_{g} Y \frac{\partial^{2} S}{\partial Y \partial G}+\rho_{r, y} \sigma_{y} \sigma_{r} Y \frac{\partial^{2} S}{\partial Y \partial R} \\
& \quad+\rho_{g, r} \sigma_{g} \sigma_{r} \frac{\partial^{2} S}{\partial G \partial R}+\frac{1}{2} \sigma_{r}^{2} \frac{\partial^{2} S}{\partial R^{2}}+\kappa_{r}\left(\mu_{r}-R\right) \frac{\partial S}{\partial R} \\
& \quad+\frac{1}{2} \sigma_{g}^{2} \frac{\partial^{2} S}{\partial G^{2}}+\kappa_{g}\left(\mu_{g}-G\right) \frac{\partial S}{\partial G}-R S+\delta Y=0
\end{aligned}
$$

subject to $S(t)<\infty$, where

$$
\lambda_{y} \equiv \sigma_{m} \sigma_{y} \rho_{m, y}
$$

is the risk premium for the systematic risk in the firm's earnings shocks, and

$$
\mu_{r} \equiv \mu_{r}^{0}-\frac{1}{\kappa_{r}} \sigma_{m} \sigma_{r} \rho_{m, r}
$$

is the long-run mean of the spot interest rate under the risk-neutral probability measure defined by the pricing kernel $M(t)$, with $\rho_{m, r}$ defined as the correlation between $\omega_{m}(t)$ and $\omega_{r}(t)$. Similarly,

$$
\mu_{g} \equiv \mu_{g}^{0}-\frac{1}{\kappa_{g}} \sigma_{m} \sigma_{g} \rho_{m, g}
$$

C Blackwell Publishing Ltd and The University of Manchester, 2005. 
is the long-run mean of the spot earnings growth rate under the risk-neutral probability measure.

The solution to PDE (7) can be shown to be

$$
S(t)=\delta \int_{0}^{\infty} s(t, G, R, Y ; \tau) \mathrm{d} \tau
$$

where $s(t, G, R, Y ; \tau)$ is the date- $t$ price of a claim that pays $Y(t+\tau)$ at future date $t+\tau$ :

$$
S(t, G, R, Y ; \tau)=Y(t) \exp [\varphi(\tau)-\rho(\tau) R(t)+\vartheta(\tau) G(t)]
$$

where

$$
\begin{aligned}
\varphi(\tau)= & -\lambda_{y} \tau+\frac{1}{2} \frac{\sigma_{r}^{2}}{\kappa_{r}^{2}}\left[\tau+\frac{1-\mathrm{e}^{-2 \kappa_{r} \tau}}{2 \kappa_{r}}-\frac{2\left(1-\mathrm{e}^{-\kappa_{r} \tau}\right)}{\kappa_{r}}\right] \\
& -\frac{\kappa_{r} \mu_{r}+\sigma_{y} \sigma_{r} \rho_{r, y}}{\kappa_{r}}\left(\tau-\frac{1-\mathrm{e}^{-\kappa_{r} \tau}}{\kappa_{r}}\right) \\
& +\frac{1}{2} \frac{\sigma_{g}^{2}}{\kappa_{g}^{2}}\left[\tau+\frac{1-\mathrm{e}^{-2 \kappa_{g} \tau}}{2 \kappa_{g}}-\frac{2}{\kappa_{g}}\left(1-\mathrm{e}^{-\kappa_{g} \tau}\right)\right] \\
& +\frac{\kappa_{g} \mu_{g}+\sigma_{y} \sigma_{g} \rho_{g, y}}{\kappa_{g}}\left(\tau-\frac{1-\mathrm{e}^{-\kappa_{g} \tau}}{\kappa_{g}}\right) \\
& -\frac{\sigma_{r} \sigma_{g} \rho_{g, r}}{\kappa_{r} \kappa_{g}}\left[\tau-\frac{1}{\kappa_{r}}\left(1-\mathrm{e}^{-\kappa_{r} \tau}\right)-\frac{1}{\kappa_{g}}\left(1-\mathrm{e}^{-\kappa_{g} \tau}\right)+\frac{1-\mathrm{e}^{-\left(\kappa_{r}+\kappa_{g}\right) \tau}}{\kappa_{r}+\kappa_{g}}\right] \\
\rho(\tau)= & \frac{1-\mathrm{e}^{-\kappa_{r} \tau}}{\kappa_{r}} \\
\vartheta(\tau)= & \frac{1-\mathrm{e}^{-\kappa_{g} \tau}}{\kappa_{g}}
\end{aligned}
$$

subject to the transversality condition that

$$
\mu_{r}-\mu_{g}>\frac{\sigma_{r}^{2}}{2 \kappa_{r}^{2}}-\frac{\sigma_{r} \sigma_{y} \rho_{r, y}}{\kappa_{r}}+\frac{\sigma_{g}^{2}}{2 \kappa_{g}^{2}}+\frac{\sigma_{g} \sigma_{y} \rho_{g, y}}{\kappa_{g}}-\frac{\sigma_{g} \sigma_{r} \rho_{g, r}}{\kappa_{g} \kappa_{r}}-\lambda_{y}
$$

Thus, the stock price is just the sum of a continuum of claims that each pays in the future an amount determined by the earnings process.

This pricing formula reflects the general valuation principle that the asset price is the sum of discounted future cash flows. Instead of a fixed schedule of payments as in the case of bonds, stocks have uncertain future cash flows, the distribution of which are determined by the earnings process of this model.

Equation (5) combined with equation (6) offers a rich yet tractable setting to model earnings. The two together imply that earnings growth is independent of earnings levels, and earnings growth depends on a current growth rate 
$G(t)$ and a long-run mean growth rate $\mu_{g}$. It turns out that such a structure plays an important role in achieving pricing accuracy, particularly because of the fact that the structure differentiates the transitory and permanent components of a firm's business, as shown in Bakshi and Chen (2001).

A source of difficulty in the BC model can be seen in equation (5), wherein earnings $Y(t)$ is modeled to follow a geometric Brownian motion. Apart from this negative earnings problem, equation (5) is a fairly good description of the earnings process. It is the analog of the most popular continuous-time model of stock prices, pioneered by Black and Scholes (1973). The geometric Brownian motion process has the property that at any level the expected return (per cent change) is the same. It is both an equilibrium requirement and an empirical fact that the stock returns are approximately independent of price levels, setting aside microstructure or liquidity issues. It also seems quite natural as a first approximation to assume that the earnings growth rate is independent of earnings levels.

The BC model has two main shortcomings. First, in principle it does not apply to any firm that has a positive probability of a zero or negative earning in the future. To the extent that this likelihood is significant, the model will be inaccurate. Second, as a practical matter of estimation, the model cannot be fitted at all to any firm which actually does have zero or negative realized earnings. Non-positive earnings observations have to be deleted or artificially treated when model prices are calculated.

Furthermore, when (positive) earnings are close to zero, the earnings growth behaves wildly. The earnings growth rate becomes infinite when earnings are arbitrarily close to zero. This means that the $\mathrm{BC}$ model price is a discontinuous function of the earnings at zero earnings point. In other words, zero earnings is the model's singularity.

In reality, zero or negative earnings are commonly observed, creating a need for a model that is well behaved with respect to this possibility. The next section provides a GEVM that accommodates the possibility of nonpositive earnings.

An important difference between earnings and stock price processes is that stock prices can never be negative by limited liability, whereas earnings can. In modeling earnings, a geometric Brownian motion process cannot change signs. Specifically, the solution to equation (5) is

$$
Y(t)=Y(0) \exp \left\{\int_{0}^{t}\left[G(\tau)-\frac{1}{2} \sigma_{y}^{2}\right] \mathrm{d} \tau+\int_{0}^{t} \sigma_{y} \mathrm{~d} \omega_{y}(\tau)\right\}
$$

If the initial earnings $Y(0)$ is assumed to be positive, all the subsequent earnings are also positive.

Another related issue concerns the current earnings growth rate $G(t)$, which in practical estimation is computed as $G(t)=Y(t+1) / Y(t)-1 . G(t)$ so defined has a singularity at $Y(t)=0$, and is not meaningful if $Y(t)$ is negative.

(C) Blackwell Publishing Ltd and The University of Manchester, 2005. 


\section{A Generalized Earnings Valuation Model}

It is easy to think of processes that allow for negative values of earnings. The challenge is to generalize the model while maintaining its desirable features. In addition to accommodating negative earnings, it is desirable to have a natural economic interpretation of the growth rate $G(t)$, and for parsimony have at most a modest number of additional model parameters. In addition, it is desirable for the model to yield a closed-form solution for the stock price.

\subsection{Decomposing Earnings}

The fact that firms with negative earnings per share can have positive market values indicates that the firm has assets which create the possibility of positive earnings in the future. ${ }^{7}$ Indeed, for some firms negative earnings for the current period may be a necessary means of achieving sustained positive earnings growth in the long run. For example, a firm may have negative earnings for several periods because of research and development expenses that will generate high future returns.

We allow for negative earnings using a seemingly minor transformation of the earnings process. Consider the formal transformation

$$
Y(t)=X(t)-y_{0}
$$

where $y_{0}$ is a positive constant. Assuming that zero is the lower bound of $X(t)$, negative $Y(t)$ is possible. We assume that $X(t)$ and its growth rate follow

$$
\begin{aligned}
& \frac{\mathrm{d} X(t)}{X(t)}=\tilde{G}(t) \mathrm{d} t+\sigma_{y} \mathrm{~d} \omega_{y}(t) \\
& \mathrm{d} \tilde{G}(t)=\kappa_{\mathrm{g}}\left[\mu_{\mathrm{g}}-\tilde{G}(t)\right] \mathrm{d} t+\sigma_{g} \mathrm{~d} \omega_{g}(t)
\end{aligned}
$$

Here $X(t)$ and $\tilde{G}(t)$ play the same role as $Y(t)$ and $G(t)$ in the Bakshi-Chen setting, although of course in practice the estimated structural parameters would be different for the transformed process than in the BC model. We refer to the above two processes as the adjusted earnings process and adjusted earnings growth process, respectively. ${ }^{8}$

Equation (15) divides the firm's earnings into adjusted earnings $X(t)$ and the negative of buffer earnings, $y_{0}$. As long as the adjusted earnings have a high enough long-run growth rate, the discounted value of the future adjusted

${ }^{7}$ For simplicity we assume that the firm never liquidates, so we do not consider any possible liquidation value of the firm.

${ }^{8}$ One practical justification for the earnings adjustment parameter $y_{0}$ is that there are accounting items such as $R \& D$ that are categorized as expenses but which in economic terms should be viewed as investments. An example is provided by the large advertising expenses of Amazon.com. Alternatively, $y_{0}$ can be interpreted as total costs in a sense to be discussed in Sections 3.3 and 4.3 . 
earnings will be greater than the discounted value of the buffer earnings, which makes a negative earnings firm have a positive stock price.

To verify that negative $Y(t)$ values are now possible, we write the solution to (16) as

$$
Y(t)=\left[Y(0)+y_{0}\right] \exp \left\{\int_{0}^{t}\left[G(\tau)-\frac{1}{2} \sigma_{y}^{2}\right] \mathrm{d} \tau+\int_{0}^{t} \sigma_{y} \mathrm{~d} \omega_{y}(\tau)\right\}-y_{0}
$$

If the first term on the right is less than (equal to) $y_{0}$, then $Y(t)$ is negative (zero). $\tilde{G}(t)$ is well-defined even when earnings $Y(t)$ are negative, as long as the adjusted earnings $X(t)$ are positive. The constant $y_{0}$ is the only additional parameter relative to the $\mathrm{BC}$ model, to be estimated from the firm's earnings data. This specification removes the singularity at $Y(t)=0$, and thereby should make the adjusted earnings process $X(t)$ behave smoothly near $Y(t)=$ 0 . The adjusted earnings and adjusted earnings growth processes also share the appealing properties of the $\mathrm{BC}$ structure.

\subsection{A Stock Valuation Formula}

The generalized earnings valuation model has a closed-form solution for a firm's stock value.

Proposition 1: Under the assumed processes (2), (3), (4), (15), (16) and (17), the equilibrium stock price is

$$
S(t)=\delta\left[Y(t)+y_{0}\right] \int_{0}^{\infty} \bar{s}[\tilde{G}(t), R(t) ; \tau] \mathrm{d} \tau-\delta y_{0} \int_{0}^{\infty} \hat{S}[R(t) ; \tau] \mathrm{d} \tau
$$

where $\left[Y(t)+y_{0}\right] \bar{s}[\tilde{G}(\mathrm{t}), R(t) ; \tau]$ is the time- $t$ price of a claim that pays $Y(t+$ $\tau)+y_{0}$ at a future date $t+\tau$,

$$
\bar{s}[\tilde{G}(t), R(t) ; \tau]=\exp [\varphi(\tau)-\rho(\tau) R(t)+\vartheta(\tau) \tilde{G}(t)]
$$

and $\hat{s}$ is the time- $t$ price of a risk-free claim that pays a constant flow of one unit forever:

$$
\hat{S}[R(t) ; \tau]=\exp [\phi(\tau)-\rho(\tau) R(t)]
$$

where

$$
\phi(\tau)=\frac{1}{2} \frac{\sigma_{r}^{2}}{\kappa_{r}^{2}}\left[\tau+\frac{1-\mathrm{e}^{-2 \kappa_{r} \tau}}{2 \kappa_{r}}-\frac{2\left(1-\mathrm{e}^{-\kappa_{r} \tau}\right)}{\kappa_{r}}\right]-\mu_{r}\left(\tau-\frac{1-\mathrm{e}^{-\kappa_{r} \tau}}{\kappa_{r}}\right)
$$

and $\varphi(\tau), \rho(\tau)$ and $\vartheta(\tau)$ are given by (10), (11) and (12), respectively, and subject to the transversality conditions

$$
\mu_{r}>\frac{1}{2} \frac{\sigma_{r}^{2}}{\kappa_{r}^{2}}
$$

(C) Blackwell Publishing Ltd and The University of Manchester, 2005. 


$$
\mu_{r}-\mu_{g}>\frac{\sigma_{r}^{2}}{2 \kappa_{r}^{2}}-\frac{\sigma_{r} \sigma_{g} \rho_{r, y}}{\kappa_{r}}+\frac{\sigma_{g}^{2}}{2 \kappa_{g}^{2}}+\frac{\sigma_{g} \sigma_{y} \rho_{g, y}}{\kappa_{g}}-\frac{\sigma_{g} \sigma_{r} \rho_{g, r}}{\kappa_{g} \kappa_{r}}-\lambda_{y}
$$

Proof: In equilibrium, the expected dividend-inclusive return of the stock in excess of the risk-free rate should be proportional to the covariance between increments of the return and the pricing-kernel process (similar to the capital asset pricing model; see Duffie, 1996)

$$
E\left[\frac{\mathrm{d} S(t)+\delta Y(t) \mathrm{d} t}{S(t)}\right]-R(t) \mathrm{d} t=-\operatorname{cov}\left[\frac{\mathrm{d} M(t)}{M(t)}, \frac{\mathrm{d} S(t)}{S(t)}\right]
$$

from which we obtain the PDE for $S(t)$ by observing that $S(t)$ is a function of $\tilde{G}(t), Y(t)$ and $R(t)$ :

$$
\begin{aligned}
& \frac{1}{2} \sigma_{y}^{2}\left(Y+y_{0}\right)^{2} \frac{\partial^{2} S}{\partial Y^{2}}+\left(\tilde{G}-\lambda_{y}\right)\left(Y+y_{0}\right) \frac{\partial S}{\partial Y}+\rho_{g, y} \sigma_{y} \sigma_{g}\left(Y+y_{0}\right) \frac{\partial^{2} S}{\partial Y \partial G} \\
& \quad+\rho_{r, y} \sigma_{y} \sigma_{r}\left(Y+y_{0}\right) \frac{\partial^{2} S}{\partial Y \partial R}+\rho_{g, r} \sigma_{g} \sigma_{r} \frac{\partial^{2} S}{\partial \tilde{G} \partial R}+\frac{1}{2} \sigma_{r}^{2} \frac{\partial^{2} S}{\partial R^{2}} \\
& \quad+\kappa_{r}\left(\mu_{r}-R\right) \frac{\partial S}{\partial R}+\frac{1}{2} \sigma_{g}^{2} \frac{\partial^{2} S}{\partial \tilde{G}^{2}}+\kappa_{g}\left(\mu_{g}-\tilde{G}\right) \frac{\partial S}{\partial \tilde{G}}-R S+\delta Y=0
\end{aligned}
$$

subject to $0<S(t)<\infty$. This equation degenerates to the BC PDE (7) if we set $y_{0}=0$.

To solve (26), conjecture the solution of the form (19). ${ }^{9}$ Then $\bar{s}$ and $\hat{s}$ satisfy

$$
\begin{aligned}
& \left(\tilde{G}-\lambda_{y}\right) \bar{s}\left(Y+y_{0}\right)+\rho_{g, y} \sigma_{g} \sigma_{y}\left(Y+y_{0}\right) \frac{\partial \bar{s}}{\partial \tilde{G}}+\rho_{r, y} \sigma_{r} \sigma_{y}\left(Y+y_{0}\right) \frac{\partial \bar{s}}{\partial R} \\
& +\rho_{g, r} \sigma_{g} \sigma_{r}\left(Y+y_{0}\right) \frac{\partial^{2} \bar{s}}{\partial \tilde{G} \partial R}+\frac{1}{2} \sigma_{r}^{2}\left(Y+y_{0}\right) \frac{\partial^{2} \bar{s}}{\partial R^{2}}-\frac{1}{2} \sigma_{r}^{2} y_{0} \frac{\partial^{2} \hat{s}}{\partial R^{2}} \\
& +\kappa_{r}\left(\mu_{r}-R\right)\left[\left(Y+y_{0}\right) \frac{\partial \bar{s}}{\partial R}-y_{0} \frac{\partial \hat{s}}{\partial R}\right]+\frac{1}{2} \sigma_{g}^{2}\left(Y+y_{0}\right) \frac{\partial^{2} \bar{s}}{\partial \tilde{G}^{2}} \\
& +\kappa_{g}\left(\mu_{g}-\tilde{G}\right)\left(Y+y_{0}\right) \frac{\partial \bar{s}}{\partial \tilde{G}}-R\left[\left(Y+y_{0}\right) \bar{s}-y_{0} \hat{s}\right]-Y \hat{s}_{\tau}=0
\end{aligned}
$$

where we have imposed the boundary conditions

$$
\begin{aligned}
& \bar{s}_{(\tau=0)}=\hat{s}_{(\tau=0)}=1 \\
& \bar{s}_{(\tau=\infty)}=\hat{s}_{(\tau=\infty)}=0
\end{aligned}
$$

${ }^{9}$ This form takes into account the fact that $\hat{s}$ should not depend on $\tilde{G}(t)$, and is important to get the correct solution. For example, the BC solution form (8) is not consistent with the PDE. 
Collecting terms by $Y+y_{0}$ and $y_{0}$ yields the PDEs for $\bar{s}$ and $\hat{s}:{ }^{10}$

$$
\begin{aligned}
& \left(\tilde{G}-\lambda_{y}-R\right) \bar{s}+\rho_{g, y} \sigma_{g} \sigma_{y} \frac{\partial \bar{s}}{\partial \tilde{G}}+\rho_{r, y} \sigma_{r} \sigma_{y} \frac{\partial \bar{s}}{\partial R}+\rho_{g, r} \sigma_{g} \sigma_{r} \frac{\partial^{2} \bar{s}}{\partial \tilde{G} \partial R} \\
& +\frac{1}{2} \sigma_{r}^{2} \frac{\partial^{2} \bar{s}}{\partial R^{2}}+\kappa_{r}\left(\mu_{r}-R\right) \frac{\partial \bar{s}}{\partial R}+\frac{1}{2} \sigma_{g}^{2} \frac{\partial^{2} \bar{s}}{\partial \tilde{G}^{2}}+\kappa_{g}\left(\mu_{g}-G\right) \frac{\partial \bar{s}}{\partial \tilde{G}}-\bar{s}_{\tau}=0
\end{aligned}
$$

and

$$
\frac{1}{2} \sigma_{r}^{2} \frac{\partial^{2} \hat{s}}{\partial R^{2}}+\kappa_{r}\left(\mu_{r}-R\right) \frac{\partial \bar{s}}{\partial R}-R \hat{s}-\hat{s}_{\tau}=0
$$

Conjecturing solutions of the forms (20) and (21), respectively, and applying the standard separation-of-variables technique, we obtain the solutions as asserted. The transversality conditions (23) and (24) are needed since we require $\hat{s}(\tau)<\infty$ and $\bar{s}(\tau)<\infty$.

It is quite intuitive that the model price is the difference between the two terms on the right-hand side of (19). The first term is the stock price of a firm with adjusted earnings $Y+y_{0}$. The second term is the stock price of a constant earnings stream $y_{0}$. The second term can be obtained from the first by setting all the terms related to the earnings growth rate $G(t)$ to zero. The firm's stock price is then simply the difference between the discounted values of the adjusted earnings and the buffer earnings. In actual implementations we need the additional requirement that $S(t)>0$, since the buffer earnings $y_{0}$ can cause negative values of $S(t)$.

\subsection{Revenues/Costs-based Stock Valuation: An Extension}

We have focused on earnings-based stock valuation, but in fact the model can be extended to more general decompositions of the earnings process. In so doing, a model that provides a better fit to the diversity of business circumstances of different firms can potentially be achieved. For example, two firms may have similar past sample histories of earnings, but these histories may have arisen by different histories of revenues and costs. The additional information in revenues and costs can potentially lead to different forecasts of future earnings, and different valuations. We therefore develop an extension of the GEVM, which we call the earnings components valuation model.

As discussed in Section 3.1, the buffer earnings parameter $y_{0}$ may be interpreted as certain costs that the firm needs to incur in order to have sustained future earnings and earnings growth. Taking this further, it would

\footnotetext{
${ }^{10}$ Alternatively, these PDEs can be obtained by observing that both $\left[Y(t+\tau)+y_{0}\right] \bar{s}[\bar{G}(t), R(t) ; \tau]$ and $\hat{s}[R(t) ; \tau]$ are contingent claims to be paid at time $t+\tau$ and therefore satisfy (25).

(C) Blackwell Publishing Ltd and The University of Manchester, 2005.
} 
be possible to view buffer earnings as costs and adjusted earnings $X(t)$ as revenues. In other words, the model differs from that in Section 3.2 in that we denote costs as $Z(t)$, and we have

$$
Y(t)=X(t)-Z(t)
$$

The level of revenues and its growth rate follow processes (16) and (17) with the interpretation that $\tilde{G}(t)$ is the instantaneous revenues growth rate. We could assume the same process for costs as well. However, to limit the number of free parameters, we will instead assume that costs $Z(t)$ follow a simple geometric Brownian motion: ${ }^{11}$

$$
\frac{\mathrm{d} Z(t)}{Z(t)}=g_{z}(t) \mathrm{d} t+\sigma_{z} \mathrm{~d} \omega_{z}(t)
$$

with constant instantaneous growth rate $g_{z}$ and variance $\sigma_{z}$.

Proposition 2: Under the assumed processes (2), (3), (4), (30), (16), (17) and (31), the equilibrium stock price $S(t)$ is a proportion $\delta$ of the difference between the discounted values of revenues and of costs:

$$
S(t)=\delta X(t) \int_{0}^{\infty} \bar{S}[\tilde{G}(t), R(t) ; \tau] \mathrm{d} \tau-\delta Z(t) \int_{0}^{\infty} \hat{S}[R(t) ; \tau] \mathrm{d} \tau
$$

Proof: Following similar steps to those in the proof of Proposition 1, we obtain the same PDE for $\bar{s}$ as before, and the PDE for $\hat{s}$ becomes

$$
\left(g_{z}-\lambda_{z}-R\right) \hat{s}+\rho_{r, z} \sigma_{r} \sigma_{z} \frac{\partial \hat{s}}{\partial R}+\frac{1}{2} \sigma_{r}^{2} \frac{\partial^{2} \hat{s}}{\partial R^{2}}+\kappa_{r}\left(\mu_{r}-R\right) \frac{\partial \bar{s}}{\partial R}-\hat{s}_{\tau}=0
$$

where $\lambda_{z}=\rho_{z, m} \sigma_{z} \sigma_{m}$ is the risk premium for the systematic risk of the firm's costs shocks. The solution to (33) is

$$
\hat{s}[R(t) ; \tau]=\exp \left[\phi_{z}(\tau)-\rho(\tau) R(t)\right]
$$

where $\rho(\tau)$ is defined as before and

$$
\begin{aligned}
\phi_{z}(\tau)= & g \tau+\frac{1}{2} \frac{\sigma_{r}^{2}}{\kappa_{r}^{2}}\left[\tau+\frac{1-\mathrm{e}^{-2 \kappa_{r} \tau}}{2 \kappa_{r}}-\frac{2\left(1-\mathrm{e}^{-\kappa_{r} \tau}\right)}{\kappa_{r}}\right] \\
& -\frac{\kappa_{r} \mu_{r}+\sigma_{h} \sigma_{r}}{\kappa_{r}}\left(\tau-\frac{1-\mathrm{e}^{-\kappa_{r} \tau}}{\kappa_{r}}\right)
\end{aligned}
$$

\footnotetext{
${ }^{11}$ If we assume a process for the growth rate of costs, then we need five more parameters to characterize costs, including three parameters as in (17), one for the correlation of costs with the interest rate and one for the risk premium of cost shocks. In addition we would need a cost forecast as one of the input variables.
} 
and where $g=g_{z}-\lambda_{z}$ and $\sigma_{h}=\sigma_{z} \rho_{r, z}$ are two additional parameters associated with costs. The transversality condition associated with $\hat{s}$ becomes

$$
\mu_{r}>g+\frac{1}{2} \frac{\sigma_{r}^{2}}{\kappa_{r}^{2}}-\frac{\sigma_{h} \sigma_{r}}{\kappa_{r}}
$$

The revenues/costs-based valuation formula (32) has inputs of the current interest rate $R(t)$, current revenues $X(t)$, the forecasted revenues growth rate (or, equivalently, one-year-ahead revenues $X(t+1)$ ) and current costs $Z(t)$. The parameter set is therefore $\left\{\mu_{g}, \kappa_{g}, \sigma_{g}, \mu_{r}, \kappa_{r}, \sigma_{r}, \lambda_{x}, \sigma_{z}, \rho_{x, z}, \delta, \sigma_{h}, g\right\}$. Compared with the earnings-based valuation model (19), the revenues/costs valuation model has one more time series input $Z(t)$ to calibrate the parameters, while having one more parameter (12 versus 11$)$. While the ratio of inputs to parameters is generally higher for the revenues/costs formula, in reality the earnings forecast data are more readily available than the revenues forecasts.

\section{Empirical Performance}

We now test the GEVM formula and the Bakshi and Chen (2001) model to evaluate whether allowing for the possibility of zero and negative earnings increases predictive power. Section 4.1 describes the model estimation method. In Section 4.2 we compare the performance of the two models. Section 4.3 examines the factors affecting the buffer earnings.

\subsection{Model Estimation Method}

For the empirical tests, we apply the GEVM formula (19) derived in Section 3.2, and compare with the BC model. Bakshi and Chen (2001) study alternative versions of their model and conclude that their 'main' model as specified in equation (8) performs far better than two special cases of equation (8) - the stochastic earnings growth model and the stochastic interest rate model. We therefore use equation (8) as our benchmark BC model price.

For predictive purposes, the out-of-sample performance of the model is of more interest than the in-sample performance. In-sample estimation uses future information that is unknown at the estimation time, and is therefore an exercise in model-fitting rather than prediction. We therefore focus exclusively on the out-of-sample performance of the main GEVM and the BC model.

Following BC, the structural parameters are estimated such that the model parameters for any particular time are estimated to minimize the sum of squared differences between the market and the model prices during the previous $T$ months. In other words, the estimation finds the value of $\Phi$ that solves

(C) Blackwell Publishing Ltd and The University of Manchester, 2005. 


$$
\min _{\Phi} \frac{1}{T} \sum_{t=1}^{T}[\hat{S}(t)-S(t)]^{2}
$$

subject to the transversality conditions (23) and (24), where $\hat{S}(t)$ denotes the observed market price at time $t$ and $T$ is the number of estimation periods.

In this section, $T$ is chosen to be 24 ( 2 years), rather than the Bakshi and Chen (2001) choice of 36 (3 years), in light of the findings of Chen and Dong (2003) that a 2-year estimation period yields better predictive power for model prices. ${ }^{12}$ The use of the stock price $S(t)$ in the objective function (38), rather than the $P / E$ ratio as in $\mathrm{BC}$, is based upon the consideration that the $P / E$ ratio is not meaningful for negative or close to zero earnings.

There are several reasons for choosing the market-implied approach as in (38) to estimating parameters rather than approaches independent of market prices. One is that the parameters can capture factors such as the firm's business, future growth opportunities and quality of management, which are missed by estimation methods that are independent of past stock prices.

Another is that the estimated structural parameters will change over time, making the model less vulnerable to mis-specification. For example, the estimated dividend payout rate $\delta$ will change from period to period, effectively relaxing the unrealistic assumption that the firm's dividend policy never changes. Finally, some parameters are not observable and can only be estimated using stock prices. Examples include the risk premium $\lambda$. Other parameters including the payout ratio $\delta$ are better estimated from stock prices than from accounting data. $\delta$ is better estimated using stock prices because, for stocks like Cisco that never pay dividends, $\delta$ (and consequently the stock price) would be zero if we were to rely on the actual dividend payout ratio. On the other hand, according to formula (19), there is a market 'implied payout ratio' such that a fraction $\delta$ of the earnings is responsible for the stock price, regardless of the actual payout ratio.

The structural parameters for the BC model include $\mu_{g}, \kappa_{g}, \sigma_{g}, \mu_{r}, \kappa_{r}, \sigma_{r}$, $\lambda_{y}, \sigma_{y}, \rho$ and $\delta$. The GEVM has the additional earnings adjustment parameter $y_{0}$. The three inputs to the model are the current year earnings $Y(t)$, the 1 -year-ahead earnings $Y(t+1)$ and the interest rate (30-year yield) $R(t) .{ }^{13}$ These inputs combined with the model parameters yield a model price.

${ }^{12}$ For the purpose of this paper, the result is not sensitive to the choice of the estimation period. Choosing a 3-year estimation period yields similar results.

${ }^{13}$ The 1 -year-ahead earnings $Y(t+1)$ becomes one of the input variables because the adjusted earnings growth rate is approximated by

$$
\tilde{G}(t)=\frac{X(t+1)}{X(t)}-1=\frac{Y(t+1)-Y(t)}{Y(t)+y_{0}}
$$

The reasons for choosing the 30 -year yield as the interest rate are that the 30 -year yield is more stock market relevant than short-term rates and that all rates should be perfectly correlated in the assumed single-factor term structure. See Bakshi and Chen (2001) for further discussion. 
The BC model price and the GEVM price are given by equations (8) and (19), respectively. The BC model price can be viewed as a special case of the GEVM price by setting $y_{0}$ to be zero in (19). A random search algorithm is applied to estimate the parameters in both models. The random search approach ensures that the parameter set $\Phi$ is closer to the global, rather than local, minimizer of (38). To improve efficiency of the estimation, the three interest rate parameters are preset at $\mu_{r}=0.07, \kappa_{r}=0.079$ and $\sigma_{r}=0.007$. These values are obtained by minimizing the sum of squared estimation errors for the S\&P 500 index. $^{14}$

\subsection{A Comparison of the BC Model and the GEVM Performance}

The previous section suggested that the GEVM should price stocks with enhanced precision and stability, because the new parameter $y_{0}$ removes the singularity of zero earnings in the $\mathrm{BC}$ model. In this section we compare the pricing performance of the $\mathrm{BC}$ model and the GEVM to determine whether this is in fact the case.

Since the BC model can only be applied to stocks with positive earnings, we focus primarily on four well-known stocks and two stock indices that have mostly positive earnings: GE, Exxon, Intel, Microsoft, the S\&P 500 index and the S\&P 400 Mid-Cap index (Mid-Cap). Among these six stocks/indices, Intel has a brief period of negative earnings, and we will see how the GEVM performs during that period. We then report briefly on a test using a broad sample of stocks, including many that experienced negative earnings.

The data for this study are from I/B/E/S US history files, which provide monthly updated earnings forecasts and contemporaneous stock prices. The sample period for each stock ends in January 1999 (1/99); depending on the stock, the beginning period varies. Table 1 presents summary statistics of the inputs data for each of the six stocks/indices to both the BC model and the GEVM. The initial 24 months of the data are not shown, since the model prices for the initial estimation period are in-sample prices.

GE, Exxon and Intel are among the earliest to enter $\mathrm{I} / \mathrm{B} / \mathrm{E} / \mathrm{S}$, with the out-of-sample period beginning from $2 / 79$. The other stocks/indices come into $\mathrm{I} / \mathrm{B} / \mathrm{E} / \mathrm{S}$ later. The stock price is most stable for the indices, followed by Exxon, GE, Microsoft and Intel, in that order. This price volatility order is matched by the volatility in earnings growth. For example, Intel's (unadjusted) earnings growth rate varies from -100 per cent to 400 per cent. This is largely because Intel experienced some periods of negative or close-to-zero earnings, which means the $\mathrm{BC}$ model will have a hard time pricing the stock during those periods.

\footnotetext{
${ }^{14}$ The approach of presetting the interest rate parameters is also employed in the $\mathrm{BC}$ study. The estimation results are not sensitive to alternative specifications of the three interest rate parameters.
}

(C) Blackwell Publishing Ltd and The University of Manchester, 2005. 
TABLE 1

Summary Statistics of the Inputs of the Stock Valuation Models

\begin{tabular}{llcccccc}
\hline & & $\begin{array}{c}\text { S\&P 500 } \\
1 / 84-\end{array}$ & $\begin{array}{c}\text { Mid-Cap } \\
3 / 85-\end{array}$ & $\begin{array}{c}\text { GE } \\
2 / 79-\end{array}$ & $\begin{array}{c}\text { Exxon } \\
2 / 79-\end{array}$ & $\begin{array}{c}\text { Intel } \\
2 / 79-\end{array}$ & $\begin{array}{c}\text { Microsoft } \\
8 / 88-\end{array}$ \\
& & $1 / 99$ & $1 / 99$ & $1 / 99$ & $1 / 99$ & $1 / 99$ & $1 / 99$ \\
& & $(N=181)$ & $(N=167)$ & $(N=240)$ & $(N=240)$ & $(N=240)$ & $(N=126)$ \\
\hline Stock & Mean & 449.76 & 553.44 & 20.58 & 25.13 & 15.94 & 25.45 \\
price $S(t)$ & Std & 259.03 & 303.69 & 21.53 & 17.16 & 26.22 & 31.61 \\
& Max & 1234.40 & 1485.06 & 96.56 & 75.56 & 139.00 & 143.81 \\
& Min & 151.40 & 199.03 & 2.89 & 6.19 & 0.80 & 1.28 \\
Current & Mean & 23.51 & 26.80 & 1.10 & 1.89 & 0.78 & 0.58 \\
earnings & Std & 8.65 & 8.79 & 0.67 & 0.55 & 1.12 & 0.52 \\
$Y(t)$ & Max & 40.64 & 43.44 & 2.72 & 3.40 & 3.97 & 1.99 \\
& Min & 13.82 & 13.63 & 0.34 & 0.78 & -0.14 & 0.07 \\
Forecasted & Mean & 26.81 & 29.53 & 1.24 & 1.90 & 0.93 & 0.70 \\
1 -year- & Std & 8.86 & 9.26 & 0.75 & 0.49 & 1.27 & 0.62 \\
ahead & Max & 44.79 & 47.64 & 3.09 & 3.11 & 4.60 & 2.45 \\
earnings & Min & 15.14 & 15.44 & 0.36 & 0.86 & 0.00 & 0.08 \\
$Y(t+1)$ & & & & & & & \\
Earnings & Mean & $15.77 \%$ & $12.55 \%$ & $13.19 \%$ & $1.46 \%$ & $34.01 \%$ & $22.38 \%$ \\
growth & Std & $11.04 \%$ & $18.75 \%$ & $8.30 \%$ & $9.60 \%$ & $60.91 \%$ & $10.26 \%$ \\
rate & & & & & & & \\
$Y(t+1) /$ & Max & $48.30 \%$ & $52.63 \%$ & $58.62 \%$ & $22.78 \%$ & $400.00 \%$ & $50.00 \%$ \\
$Y(t)-1$ & Min & $1.15 \%$ & $-46.94 \%$ & $2.47 \%$ & $-31.12 \%$ & $-100.00 \%$ & $-2.33 \%$ \\
30 -year & Mean & $8.92 \%$ & $7.70 \%$ & $8.92 \%$ & $8.92 \%$ & $8.92 \%$ & $7.31 \%$ \\
yield & Std & $1.76 \%$ & $1.31 \%$ & $2.31 \%$ & $2.31 \%$ & $2.31 \%$ & $1.05 \%$ \\
$R(t)$ & Max & $13.64 \%$ & $11.84 \%$ & $14.87 \%$ & $14.87 \%$ & $14.87 \%$ & $9.31 \%$ \\
& Min & $4.90 \%$ & $4.90 \%$ & $4.90 \%$ & $4.90 \%$ & $4.90 \%$ & $4.90 \%$ \\
\hline
\end{tabular}

Notes: This table shows descriptive statistics for the inputs of the BC model and the GEVM. The BC model price and the GEVM price are given by formulas (8) and (19), respectively. For both models, the inputs for computing the time- $t$ model price include the current earnings $Y(t)$, the forecasted 1-year-ahead earnings $Y(t$ +1 ) and the interest rate (30-year yield) $R(t)$. At time $t$, the model parameters are estimated to minimize the sum of squared differences between the market prices and the model prices during the previous 24 months. Only the out-of-sample period data are shown (i.e. this table does not include the initial 2 years' data of I/B/E/S coverage for each stock). $N$ is the number of observations.

${ }^{a}$ Earnings growth rate applies only to positive $Y(t)$ observations.

The model prices over the sample period for the $\mathrm{BC}$ model and the GEVM for each stock are plotted in Fig. 1, along with the corresponding market prices. It is evident that the GEVM price tracks the market price much more closely than the BC model price does. Furthermore, BC prices seems to have volatility unrelated to market prices for all six stocks/indices; this excess model volatility does not seem to be a problem for the GEVM. The BC model price for Intel experiences some jumps toward the end of 1987, due to the negative-earnings problem just mentioned. The other BC model price jump that occurred more recently for Intel is due to the random search algorithm in the estimation of the parameters, which can yield large pricing errors for the BC model. In other words, the BC model is badly specified in some circumstances. 

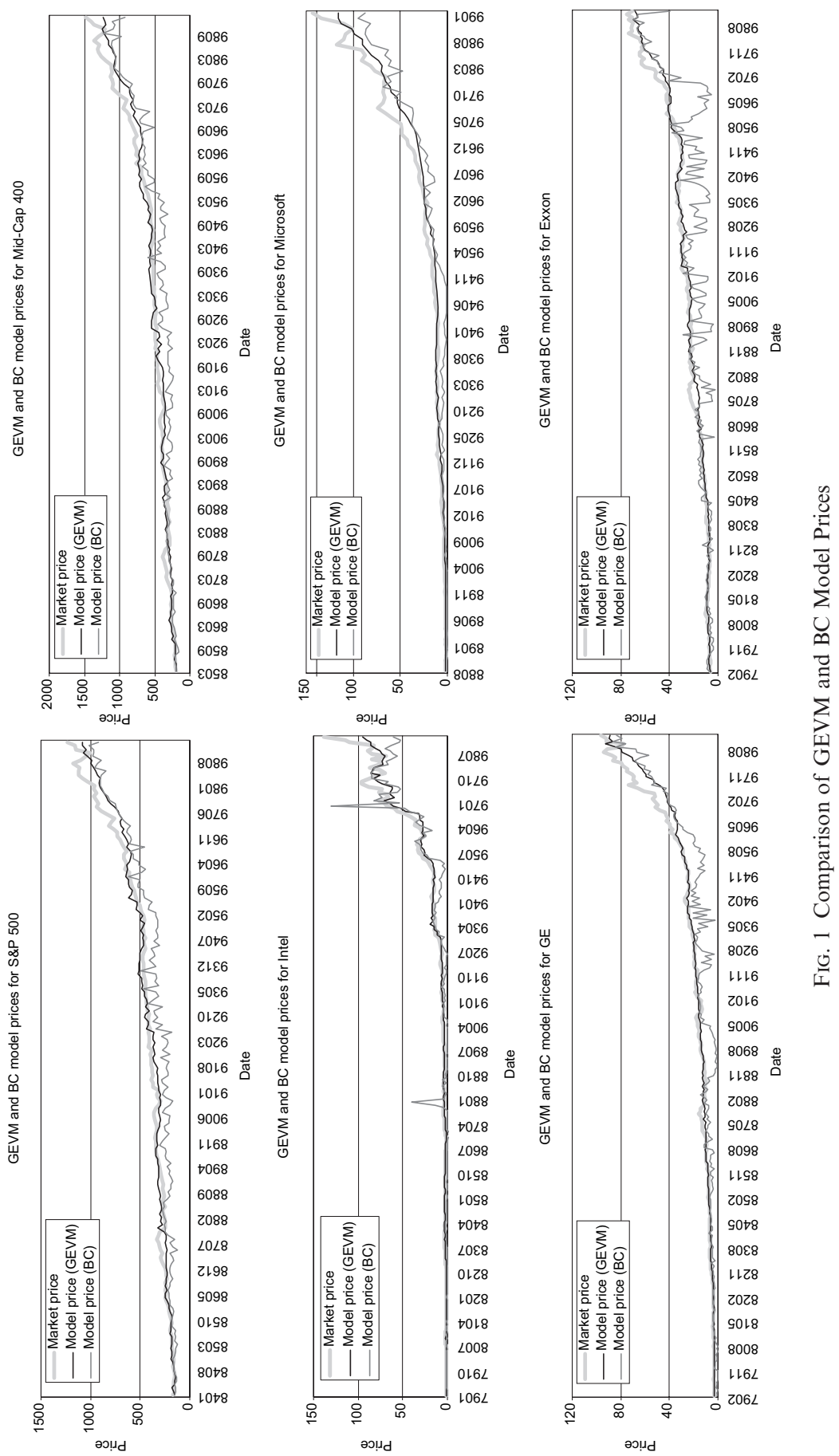

(C) Blackwell Publishing Ltd and The University of Manchester, 2005. 
It is also evident that the $\mathrm{BC}$ model price is almost always lower than the market price during the more recent years of booming stock market. In contrast, the GEVM tracks the market price remarkably well even during the negative-earnings period, showing that $y_{0}$ successfully solves the negativeearnings modeling problem for Intel.

Table 2 documents the dollar and percentage mispricing for each stock and for each model. The dollar mispricing is defined as the difference between the market and the model prices. The percentage mispricing is defined as the dollar mispricing over the model price. To minimize the effect of bad parameter estimation, the model price is restricted to be within 0.4 to 5 times of the market price, for both models. We use the terms 'underpricing' or 'undervaluation' to refer to stocks with negative mispricing (i.e. market price below model price). This language is from the perspective of the market inefficiently making a valuation error when the market price differs from the model price. Of course, part or all of this deviation may actually be due to error on the part of the model rather than the market. ${ }^{15}$

Table 2 indicates that the pricing error of the GEVM is much smaller than the $\mathrm{BC}$ model for all the stocks and indices examined; the mean mispricing numbers are closer to zero for the GEVM. The GEVM price is less volatile (the standard deviation and the range of the mispricing numbers are smaller). The $\mathrm{BC}$ model does a better job pricing indices (percentage mispricing around 30 per cent) than individual stocks (mispricing around 70 per cent), presumably because indices tend to have lower earnings volatility, so that the proximity to zero of the earnings is less of a problem for indices.

To examine what helps the GEVM achieve its superior pricing performance over the $\mathrm{BC}$ model, Table 3 reports the mean and standard deviation of the time series parameter estimates for each stock for both models. Since a random parameter search algorithm is used, the parameters shift from time to time for each stock, but the estimates appear to be reasonably stable and meaningful. For example, the long-run growth rate for the high-tech stocks Intel and Microsoft is close to 12 per cent, while it is lower (about 7 per cent) for GE, and even lower for Exxon ( 2 per cent), reflecting the nature of the firm's growth opportunities.

Somewhat surprisingly, all the seven common parameters for both models are similar in mean and standard deviation for the two models for every stock. Therefore the key difference must lie in the earnings adjustment parameter $y_{0}$. This parameter is effectively constrained to be fixed at zero for the $\mathrm{BC}$ model, while the estimated $y_{0}$ for the GEVM is statistically and economically different from zero, yielding smaller SSEs (square root of sum of

\footnotetext{
${ }^{15}$ If the model price is closer to long-term fundamental value than the market price, we would expect mispricing to be mean-reverting, a property discussed below. However, a more direct test is to examine whether model mispricing predicts future abnormal stock returns; see Chen and Dong (2003).
} 


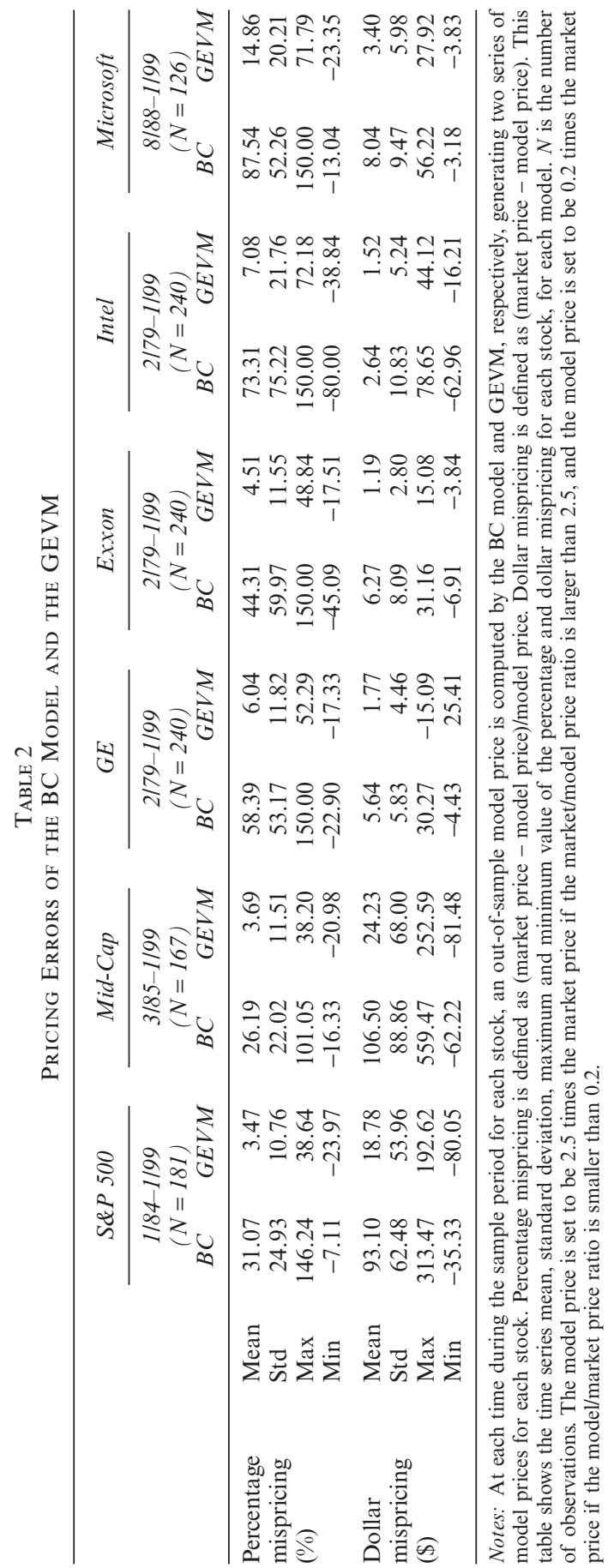

(C) Blackwell Publishing Ltd and The University of Manchester, 2005. 


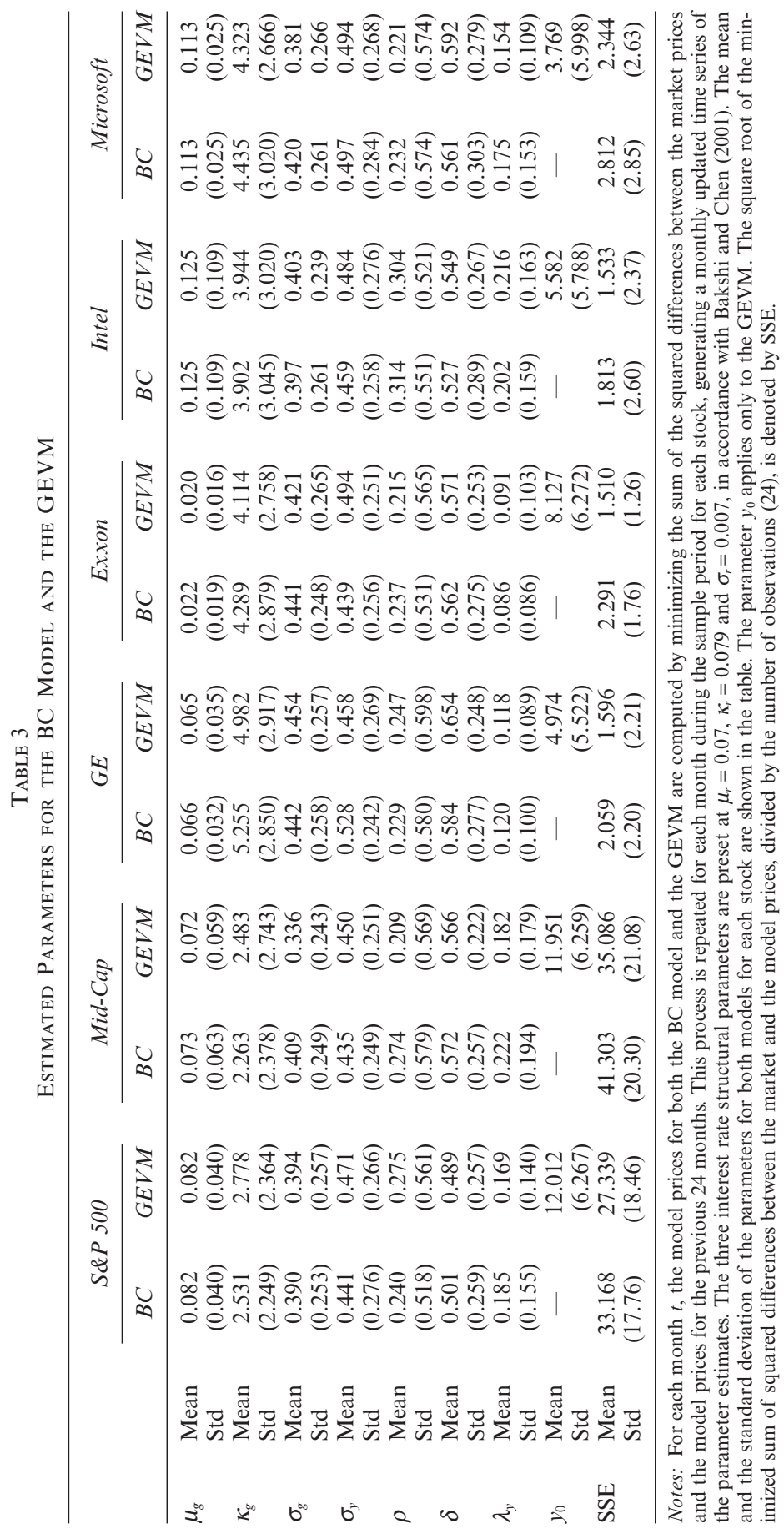


TABLE 4

Correlation Matrix of Percentage Mispricing among Assets

\begin{tabular}{|c|c|c|c|c|c|c|}
\hline & $S \& P 500$ & Mid-Cap & $G E$ & Exxon & Intel & Microsoft \\
\hline \multicolumn{7}{|c|}{ (A) Correlation of the $B C$ model mispricing } \\
\hline S\&P 500 & 1.00 & & & & & \\
\hline Mid-Cap & 0.46 & 1.00 & & & & \\
\hline GE & 0.23 & 0.02 & 1.00 & & & \\
\hline Exxon & 0.16 & 0.13 & 0.11 & 1.00 & & \\
\hline Intel & 0.10 & 0.24 & 0.12 & -0.13 & 1.00 & \\
\hline Microsoft & 0.25 & 0.15 & 0.41 & 0.18 & 0.47 & 1.00 \\
\hline \multicolumn{7}{|c|}{ (B) Correlation of the GEVM mispricing } \\
\hline S\&P 500 & 1.00 & & & & & \\
\hline Mid-Cap & 0.92 & 1.00 & & & & \\
\hline GE & 0.71 & 0.72 & 1.00 & & & \\
\hline Exxon & 0.65 & 0.65 & 0.50 & 1.00 & & \\
\hline Intel & 0.56 & 0.57 & 0.52 & 0.46 & 1.00 & \\
\hline Microsoft & 0.60 & 0.52 & 0.37 & 0.37 & 0.59 & 1.00 \\
\hline
\end{tabular}

Notes: This table shows the contemporaneous correlation of the percentage mispricing of the six stocks under the $\mathrm{BC}$ model and the GEVM. Percentage mispricing is defined as (market price - model price)/model price. Since the sample periods for the stocks differ, the correlation is based on the overlapping period for each pair of stocks.

squared errors divided by 24) for the GEVM. For instance, the mean estimated $y_{0}$ is close to 12 for the two indices, with standard deviation being about 6 . This confirms that the model does need $y_{0}$ as a buffer in the earnings and earnings growth processes, and that $y_{0}$ is crucial in bringing in stability and precision to the model price.

In the BC study, different stocks' mispricing levels are not always positively correlated. BC conclude that some stocks are bargains (underpriced by the market) even when other stocks are overpriced. Table 4 presents the Pearson correlation matrix of contemporaneous percentage mispricing among the six stocks/indices. The conclusions from the $\mathrm{BC}$ model and the GEVM are quite different. While the correlations under the BC model tend to be small and sometimes negative (the -0.13 correlation between Intel and Exxon is significant at the 5 per cent level), the GEVM says that the correlations in mispricing for these six stocks/indices are much higher. In other words, there are significant co-movements in the mispricing across stocks.

Because of the high correlation of mispricing among stocks, undervaluation or overvaluation of factors or of the entire market will tend to occur at different times. Nevertheless, some individual stocks will tend to be more of a 'bargain' than others. We can rank stocks on a relative basis according to their mispricings. Given the evidence that the GEVM has higher precision than the $\mathrm{BC}$ model, the lower correlation in $\mathrm{BC}$ mispricing across stocks is probably due to noise in the $\mathrm{BC}$ model prices.

(C) Blackwell Publishing Ltd and The University of Manchester, 2005. 
Finally, BC document that stock mispricing tends to be mean-reverting, i.e. underpriced stocks tend to become less underpriced as time elapses, and overpriced stocks tend to become less overpriced. To compare the meanreversion tendency property for the two models, Fig. 2 plots the autocorrelation of percentage mispricing at different lags for each stock under both models. The mispricing under the GEVM presents clearer and stronger patterns of mean-reversion. The autocorrelation of mispricing falls from around 0.8 to zero in about 12 months and becomes negative afterwards for all the six stocks/indices. The autocorrelation under the $\mathrm{BC}$ model presents noisier and generally slower mean-reversion. Since mean-reversion is a desirable property of a measure of market mispricing, this evidence is more supportive of the GEVM than the BC model.

For comparison between the BC model and the GEVM, the above study has focused on the six well-known stocks/indices that generally do not have the negative-earnings problem, yet the GEVM performs considerably better than the BC model. In other studies, the GEVM has been applied to find model prices for a wider range of stocks, including many with negative earnings. ${ }^{16}$ The GEVM can handle stocks with negative earnings just as well as other stocks, and it has been shown that the GEVM price possesses significant return predictive power, even after controlling for the known factors such as firm size, book-market ratio and momentum.

\subsection{What Factors Affect the Buffer Earnings?}

The previous section shows that the buffer earnings $y_{0}$ plays a crucial role in achieving superior pricing performance. In this section, we will investigate what factors are related to $y_{0}$ in order to provide insight into its economic meaning. As discussed above, $y_{0}$ is one of the model parameters that are estimated from the earnings and market prices data. It would be interesting to see whether $y_{0}$ is related to some observable variables. As discussed in Section $3, y_{0}$ may be interpreted as part of the total costs (or, in the extreme case as in Section 3.3, the total costs).

In order to do this, we use a much larger data sample that contains all the I/B/E/S-covered stocks which are also listed in CRSP and Compustat. The market stock prices are cross-checked across the I/B/E/S and CRSP data sets to ensure accuracy. Table 5 shows the number of stocks each year in this full sample. The number of stocks each year increases steadily over time, with an average of 1090 stocks each year.

Table 6 provides summary statistics for the variables of interest, including research and development expenditure (R\&D), advertisement expenses (ADV), depreciation expenses (DEPRE), total costs (COST) and current

\footnotetext{
${ }^{16}$ These studies include Chang (1999), Jindra (2000), Chen and Jindra (2001), Brown and Cliff (2002), Chen and Dong (2003).
}

(C) Blackwell Publishing Ltd and The University of Manchester, 2005. 

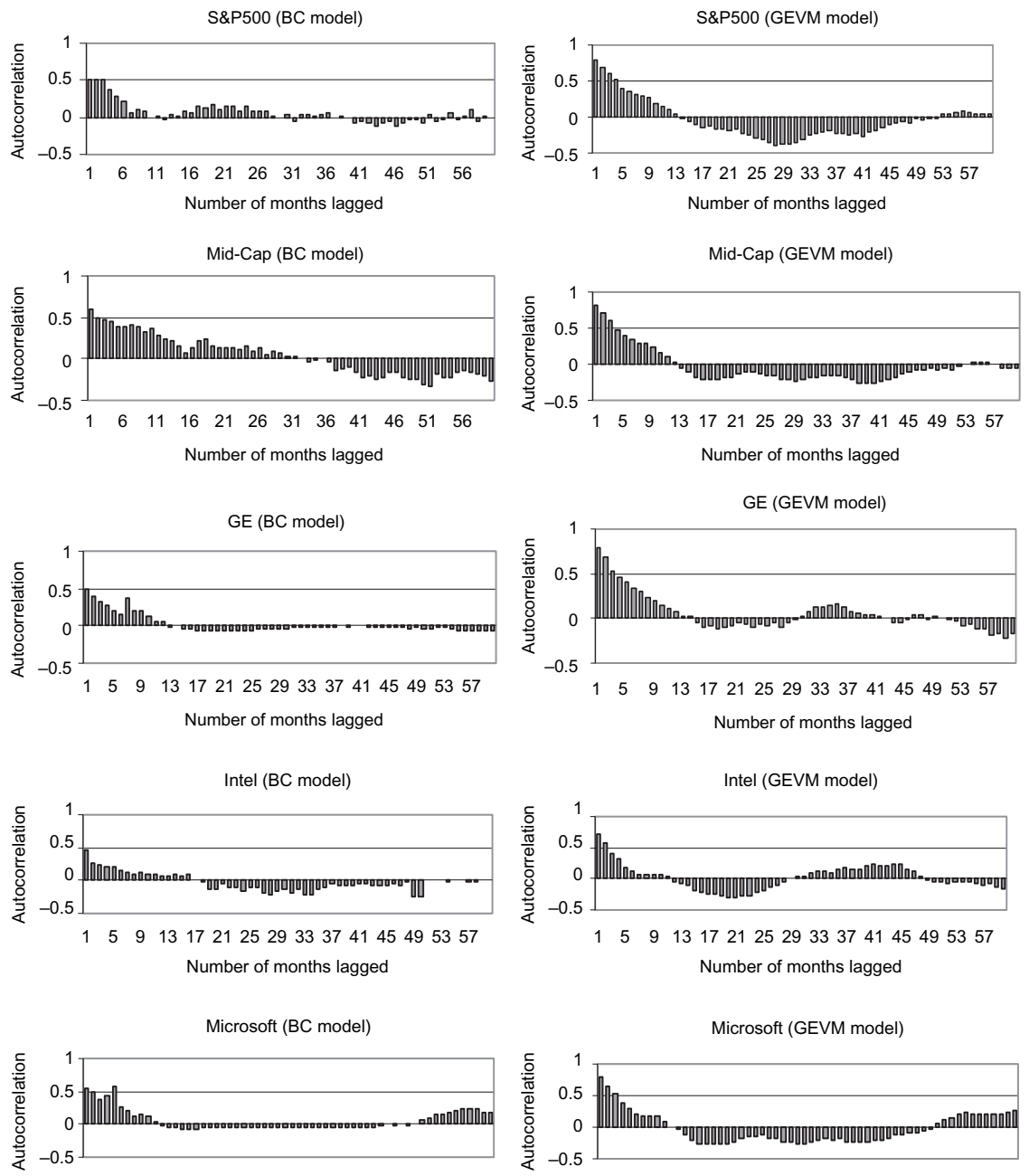

$\begin{array}{lllllllllllllll}5 & 5 & 9 & 13 & 17 & 21 & 25 & 29 & 33 & 37 & 41 & 45 & 49 & 53 & 57\end{array}$

Number of months lagged

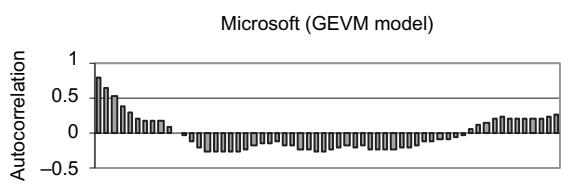

Exxon (BC model)
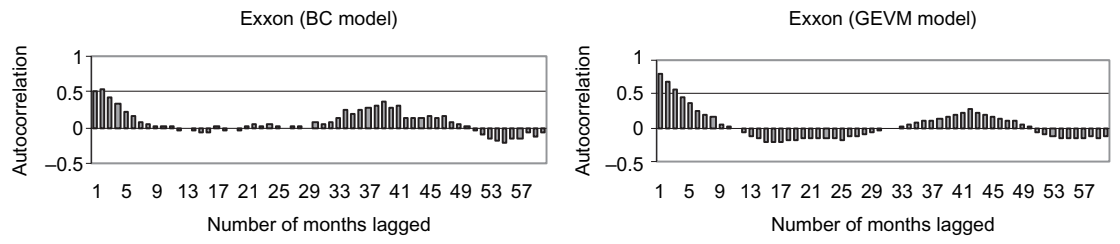

FIg. 2 Autocorrelation of Percentage Mispricing 
TABLE 5

Number of Stocks in the Whole Sample

\begin{tabular}{lc}
\hline Year & No. of stocks \\
\hline 1979 & 438 \\
1980 & 566 \\
1981 & 608 \\
1982 & 622 \\
1983 & 672 \\
1984 & 731 \\
1985 & 796 \\
1986 & 881 \\
1987 & 915 \\
1988 & 977 \\
1989 & 1112 \\
1990 & 1203 \\
1991 & 1257 \\
1992 & 1349 \\
1993 & 1464 \\
1995 & 1733 \\
1996 & 1975 \\
Mean & 2316 \\
\hline
\end{tabular}

Notes: The stocks in the whole sample are selected from the intersection of three databases: CRSP, Compustat and I/B/E/S. The data are doublechecked so that stock prices from CRSP and I/B/E/S match. The original sample from the selection process starts in 1977. As the model estimation requires two years of prior data for each stock, the final sample starts from January 1979, so that the model price for each stock and for every month is determined out of the parameter-estimation sample (out-ofsample model price).

earnings (EARN). All these accounting data are obtained from the annual Compustat files. ${ }^{17}$

Because the accounting variables have high serial correlations, and because mispricing may not be directly comparable across time, a pooled regression over all sample periods is not appropriate here. As discussed in the preceding section, mispricing tends to be correlated at any given time, and a certain level of mispricing may mean relative underpricing of the stock at a time of overall market overvaluation, while the same level of mispricing may mean relative overpricing at a time of overall market undervaluation. We therefore examine relative mispricing cross-sectionally rather than across time. To do so we employ Fama-MacBeth regressions which make crosssectional comparisons of mispricing and accounting variables.

Table 7 presents the Fama-MacBeth regression results. Since most of these accounting variables are also positively correlated, in several cases sub-

\footnotetext{
${ }^{17} \mathrm{R} \& \mathrm{D}$ is annual data item 46; advertisement is annual data item 45; depreciation is annual data item 103; total costs is sales (data item 12) minus current earnings (data item 172). 
TABLE 6

Summary Statistics of Variables Affecting Buffer Earnings

\begin{tabular}{lccccccc}
\hline $\begin{array}{l}\text { Descriptive } \\
\text { statistics }\end{array}$ & $\begin{array}{c}\text { MISP } \\
(\%)\end{array}$ & $\begin{array}{c}y_{0} \\
\text { (\$/share) }\end{array}$ & $\begin{array}{c}\text { R\&D } \\
\text { (\$/share) }\end{array}$ & $\begin{array}{c}\text { ADV } \\
\text { (\$/share) }\end{array}$ & $\begin{array}{c}\text { DEPRE } \\
\text { (\$/share) }\end{array}$ & $\begin{array}{c}\text { COST } \\
\text { (\$/share) }\end{array}$ & $\begin{array}{c}\text { EARN } \\
\text { (\$/share) }\end{array}$ \\
\hline $\begin{array}{l}\text { (A) Univariate } \\
\text { Statistics }\end{array}$ for each variable & & & & & \\
Mean & 3.23 & 8.69 & 0.37 & 0.24 & 0.57 & 25.43 & 1.20 \\
Max & 149.95 & 39.93 & 1932.5 & 851.4 & 186.9 & 8694.8 & 1655.1 \\
75 th & 12.97 & 14.48 & 0.28 & 0.07 & 0.59 & 29.14 & 1.66 \\
$\quad$ percentile & & & & & & & \\
$\begin{array}{l}\text { Median } \\
\text { 25th }\end{array}$ & 1.65 & 8.30 & 0.00 & 0.00 & 0.03 & 14.68 & 0.86 \\
$\quad$ percentile & -8.78 & 2.36 & 0.00 & 0.00 & 0.00 & 7.11 & 0.34 \\
$\begin{array}{l}\text { Min } \\
\quad-74.81\end{array}$ & 0.00 & 0.00 & 0.00 & 0.00 & -7.11 & -33.64
\end{tabular}

(B) Correlation matrix of the variables

MISP $\quad 1.000$

$\begin{array}{lll}y_{0} & -0.077 & 1.000\end{array}$

(0.003) $\quad 0.005-1.000$

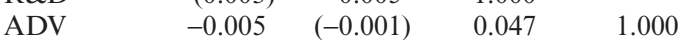

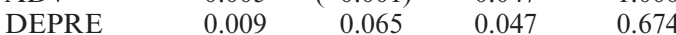

$\begin{array}{lllll}\text { COST } \quad(-0.002) & 0.055 & 0.685 & 0.538\end{array}$

EARN $\quad 0.004 \quad 0.006 \quad 0.952$

$0.212 \quad 0.217$

1.000

0.795

1.000

Notes: This table reports summary statistics for the GEVM-determined mispricing (MISP $=$ market $/$ model pric -1$)$, buffer earnings $\left(y_{0}\right)$, research and development expenditures (R\&D), advertising expenses (ADV), depreciation expenses (DEPRE), total costs (COST) and current earnings (EARN), for the sample period January 1979-December 1996. All accounting data are obtained from the annual Compustat. All data items are on a per share basis. For the correlation matrix in (B), all entries are statistically significant at the 5 per cent level except for those in parentheses.

stantially so Table 6, part (B), we primarily examine the independent variables individually in univariate tests. It is evident from Table 6, part (A), that $y_{0}$ is positively related to all types of expenses (and earnings). Firms with high expenses tend to have high $y_{0}$. It should be emphasized that the model estimation does not use these expenses as inputs. Thus, this relationship is not just a mechanical consequence of the estimation procedure. In addition, this finding is not sensitive to whether expenses are measured relative to market capitalization rather than on a per share basis.

Finally, Table 7, part (B), indicates that both $y_{0}$ and $\mathrm{R} \& \mathrm{D}$ are negatively correlated with the level of mispricing. In other words, stocks with high $y_{0}$ tend to be undervalued by the market. Furthermore, stocks with high R\&D expenditure per share tend to be undervalued. In results not reported in the table, mispricing is also negatively correlated with advertising and depreciation if these expenses are measured against market value of equity. These findings are consistent with those of Chan et al. (2000), who document that firms with high $R \& D$ and advertising expenditures tend to experience high subsequent abnormal stock returns (if $\mathrm{R} \& \mathrm{D}$ and advertising are scaled by market capitalization). 


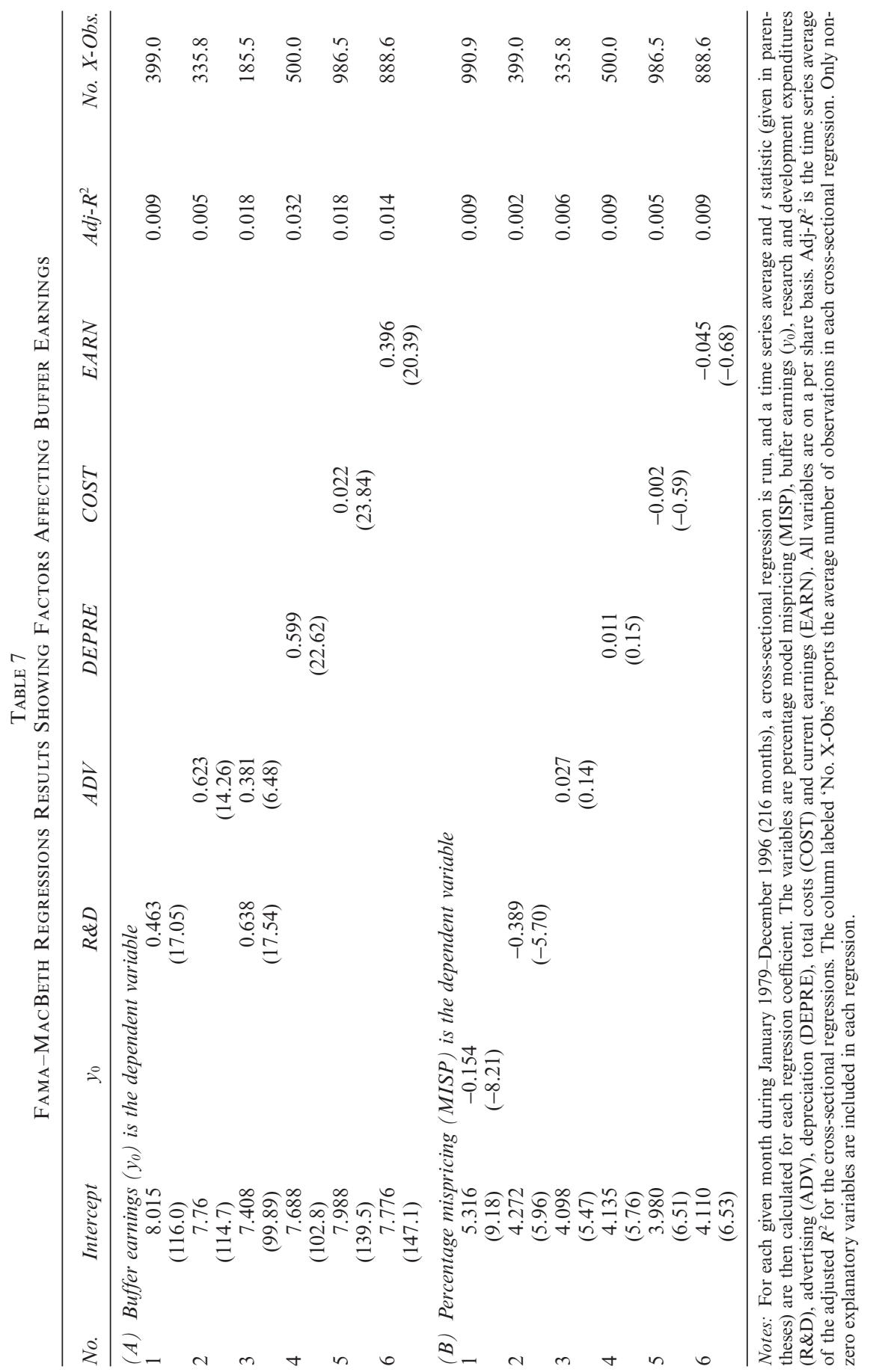

(C) Blackwell Publishing Ltd and The University of Manchester, 2005. 


\section{Conclusion}

This paper introduces an earnings-based stock valuation model which generalizes the model of Bakshi and Chen (2001) to allow for stocks that have a positive probability of zero or negative earnings per share. By adding one new earnings-adjustment parameter, buffer earnings, and introducing adjusted earnings and adjusted earnings growth concepts to the $\mathrm{BC}$ model, the GEVM inherits the appealing properties of the $\mathrm{BC}$ model but prices stocks with much improved flexibility and precision.

The GEVM removes the BC model's singularity at zero earnings point, and therefore performance is especially improved for stocks with earnings that are close to zero. Because the buffer earnings tend to smooth out earnings growth rate, the GEVM also improves pricing performance for firms with more volatile earnings. We find that the empirical predictive performance of the GEVM is superior to that of the BC model, with smaller pricing errors, greater stability and stronger mean-reversion of the model mispricing. We also find that the buffer earnings variable, which is crucial for the GEVM's superior pricing performance, is positively related to a variety of the firm's expense variables (even though it is not estimated directly from these accounting variables).

The GEVM as developed here provides a general means of pricing stocks based upon current earnings, forecasted future earnings and interest rates data. The relaxation of the negative-earnings condition therefore makes the GEVM particularly attractive for large-scale asset pricing or corporate event studies. The recent work of Chen and Dong (2003) is an example of such study.

We also develop an extended version of the GEVM which separately models stochastic revenue and cost processes, instead of a single combined earnings process. The valuation formula is broadly similar in form, but has more parameters and requires more inputs than the earnings approach. An advantage of the revenues/costs approach is there are more input variables relative to the number of parameters to be estimated, but data availability and accuracy may be greater for the earnings approach. Therefore which approach yields better performance is an open empirical question.

One direction for extending the model is to incorporate the possibility of bankruptcy and stochastic liquidation value. In addition, the assumed Vasicek term structure of interest rates and the linear assumption of the dividend payout are both approximations. Incorporating more realistic term structure and dividend assumptions could provide an even more accurate predictive model. A challenge for future research is to incorporate richer structures to the model while retaining empirical implementability.

There has been a steadily growing literature of behavioral finance that builds on the premise of investor irrationality and market misvaluation (see the surveys of Hirshleifer (2001) and Baker et al. (2004)). Critical for many 
lines of research in behavioral finance is the availability of a measure of market misvaluation. Stock valuation models can be applied to measure the mispricing levels of individual stocks as well as the aggregate market. The residual income model has been employed to measure stock market misvaluation to test return predictability in asset pricing (e.g. Frankel and Lee, 1998; Lee et al., 1999; Ali et al., 2003) and to test behavioral finance theories in corporate finance (e.g. Dong et al., 2005; Rhodes-Kropf et al., 2005). Given the potential advantages of the GEVM over the residual income model, it may be fruitful to apply the GEVM in similar contexts. These and other directions provide rich avenues for future research.

\section{REFERENCES}

Ali, A., Hwang, L.-S. and Trombley, M. A. (2003). 'Residual-income-based Valuation Predicts Future Returns: Evidence on Mispricing versus Risk Explanations', Accounting Review, Vol. 78, pp. 377-396.

Ang, A. and Liu, J. (2001). 'A General Affine Earnings Valuation Model', Review of Accounting Studies, Vol. 6, pp. 397-425.

Baker, M., Ruback, R. S. and Wurgler, J. (2004). 'Behavioral Corporate Finance: a Survey', Unpublished working paper, New York University.

Bakshi, G. and Chen, Z. (2001). 'Stock Valuation in Dynamic Economies', Unpublished working paper, Yale University and University of Maryland.

Bakshi, G. and Ju, N. (2002). 'Book Values, Earnings, and Market Valuations', Unpublished working paper, University of Maryland.

Berk, J., Green, R. and Naik, V. (1999). 'Optimal Investment, Growth Options and Security Returns', Journal of Finance, Vol. 54, pp. 1553-1607.

Black, F. and Scholes, M. (1973). 'The Pricing of Options and Corporate Liabilities', Journal of Political Economy, Vol. 81, pp. 637-659.

Brennan, M. and Schwartz, E. (1979). 'A Continuous Time Approach to the Pricing of Bonds', Journal of Banking and Finance, Vol. 3, pp. 133-156.

Brown, G. and Cliff, M. (2002). 'Sentiment and the Stock Market', Unpublished working paper, University of North Carolina.

Chan, K. C., Lakonishok, J. and Sougiannis, T. (2000). 'The Stock Market Valuation of Research and Development Expenditures', Journal of Finance, Vol. 56, pp. 2431-2451.

Chang, C. (1999). 'A Re-examination of Mergers Using a Stock Valuation Model', Unpublished working paper, Ohio State University.

Chen, Z. and Dong, M. (2003). 'Stock Valuation and Investment Strategies', Unpublished working paper, Yale University and York University.

Chen, Z. and Jindra, J. (2001). 'A Valuation Study of Stock-market Seasonality and Firm Size', Unpublished working paper, Yale University.

Cox, J., Ingersoll, J. and Ross, S. (1985). 'A Theory of the Term Structure of Interest Rates', Econometrica, Vol. 53, pp. 385-408.

Dong, M., Hirshleifer, D., Richardson, S. and Teoh, S. H. (2005). 'Does Investor Misvaluation Drive the Takeover Market?', Journal of Finance, forthcoming.

Duffie, D. (1996). Dynamic Asset Pricing Theory, 2nd edn, Princeton, NJ, Princeton University Press.

Frankel, R. and Lee, C. (1998). 'Accounting Valuation, Market Expectation, and the Cross-sectional Stock Returns-Heuristics and Biases', Journal of Accounting and Economics, Vol. 25, pp. $214-412$. 
Gordon, M. (1962). The Investment, Financing and Valuation of the Corporation, Homewood, IL, Irwin.

Harrison, M. and Kreps, D. (1979). 'Martingales and Arbitrage in Multiperiod Security Markets', Journal of Economic Theory, Vol. 20, pp. 381-408.

Hirshleifer, D. (2001). 'Investor Psychology and Asset Pricing', Journal of Finance, Vol. 64, pp. 1533-1597.

Jindra, J. (2000). 'Seasoned Equity Offerings, Overvaluation, and Timing', Unpublished working paper, Ohio State University.

Kothari, S. P., Lewellen, J. and Warner, J. (2005). 'Stock Returns, Aggregate Earnings Surprises, and Behavioral Finance', Journal of Financial Economics, forthcoming.

Lee, C., Myers, J. and Swaminathan, B. (1999). 'What is the Intrinsic Value of the Dow?', Journal of Finance, Vol. 54, pp. 1693-1741.

Lintner, J. (1956). 'Distribution of Incomes of Corporations among Dividends, Retained Earnings, and Taxes', American Economic Review, Vol. 76, pp. 97-118.

Longstaff, F. and Schwartz, E. (1992). 'Interest Rate Volatility and the Term Structure: a Two-factor General Equilibrium Model', Journal of Finance, Vol. 47, pp. $1259-1282$.

Ohlson, J. A. (1995). 'Earnings, Book Values, and Dividends in Equity Valuation', Contemporary Accounting Research, Vol. 11, pp. 661-687.

Penman, S. and Sougiannis, T. (1998). 'A Comparison of Dividend, Cash Flow, and Earnings Approach to Equity Valuation', Contemporary Accounting Research, Vol. 15, pp. 343-383.

Rhodes-Kropf, M., Robinson, D. and Viswanathan, S. (2005). 'Valuation Waves and Merger Activity: the Empirical Evidence', Journal of Financial Economics, forthcoming.

Ritter, J. and Warr, R. (2002). 'The Decline of Inflation and the Bull Market of 1982-1999', Journal of Financial and Quantitative Analysis, Vol. 37, pp. 29-61.

Vasicek, O. (1977). 'An Equilibrium Characterization of the Term Structure', Journal of Financial Economics, Vol. 5, pp. 177-188. 